\title{
Theorems on the Renormalization Group Evolution of Quark Yukawa Couplings and CKM Matrix
}

\author{
P. Kielanowski ${ }^{1,2}$, S.R. Juárez W. ${ }^{3}$ and J.G. Mora H. ${ }^{2}$ \\ ${ }^{1}$ Institute of Theoretical Physics, University of Biatystok, Poland \\ 2 Departamento de Física, CINVESTAV, México \\ ${ }^{3}$ Escuela Superior de Física y Matemáticas, IPN, México
}

\begin{abstract}
We analyze the two loop renormalization group equations in the standard model and its extensions for the coupling constants and the quark Yukawa couplings. The key point of our analysis is the observed hierarchy of the quark masses and the CKM matrix. For the one loop evolution we find the explicit solution for the evolution of the Yukawa couplings and show the following: 1. the CKM matrix depends on the energy on only one function of energy; 2 . the ratios of the down quark masses depend on the energy through the same function as the CKM matrix; 3. the diagonalizing matrices of the biunitary transformation of the up quarks are energy independent. Next we give the explicit form of the two loop corrections to the evolution of the quark Yukawa couplings and show that the relative corrections are of the order $\lambda^{4}$ for the quark Yukawa couplings and of the order $\lambda^{5}$ for the CKM matrix. Finally we give the equations of the one loop evolution of the squares of the matrix elements of the CKM matrix and their explicit solution.
\end{abstract}

05.10.Cc,11.10.Hi,12.15.Hh

\section{INTRODUCTION}

The problem of the Renormalization Group EVolution (RGEV) of the Quark Yukawa Couplings (QYC) and the Cabibbo-Kobayashi-Maskawa matrix (CKM) has been studied in many earlier papers, Refs. 11 3. Despite the fact that many, both analytical end numerical results have been obtained on the evolution of the QYC and CKM matrix there is no single reference where some general properties of the evolution have been discussed. This paper is aimed to fill this gap and it discusses the general properties of the evolution of the CKM matrix and quark masses that follow from the hierarchical structure of the QYC.

The information on the properties of the QYC come from the experimental values of the quark masses and the CKM matrix. Both the quark masses and the CKM matrix show the hierarchical structure with the parameter $\lambda \approx \sin \theta_{C} \approx 0.22$. This hierarchical structure must also be present in the QYC and is used to derive some conditions for the unknown QYC, Refs. [- 4 which reduce the number of parameters of the standard model.

In this paper we systematically investigate the influence of the hierarchical structure on the evolution of the CKM matrix and quark masses by constructing the exact solution of the one loop RGEV equations compatible with the observed hierarchy and then considering the two loop RGEV equations with the next order corrections in $\lambda$.

The most important result that we derive is that the CKM evolution depends only on one parameter which is a suitable integral that depends on the model (Standard Model (SM), Minimal Supersymmetric Standard Model (MSSM) and Double Higgs Model (DHM)). The corrections to the one loop evolution of the CKM matrix are of the relative order $\lambda^{5}$. We next show that the evolution of the ratios of the down quark masses $m_{d} / m_{s}$ and $m_{s} / m_{b}$ depends on the same parameter as the CKM matrix. The evolution of the up quark masses is different. Here the quark masses $m_{u}, m_{c}$ depend linearly on the corresponding initial values and their ratio $m_{u} / m_{c}$ is constant 5 while the dependence for $m_{t}$ is non linear.

The organization of the paper is the following. In Section II we introduce the notation and the essential experimental facts. Afterwards we discuss the RGEV equations up to two loops and their approximate form compatible with the observed hierarchy. This gives the basis for the derivation in Section III of the exact solutions of the one loop RGEV equations. We also present there the perturbative scheme for the study of the corrections coming from the higher order terms in $\lambda$ and the two loop contributions. In Section IV we discuss the properties of the solutions derived earlier and their physical importance. In Section $\mathrm{V}$ we derive the approximate evolution equations for the squares of the absolute values of the CKM matrix elements and give their explicit solution. In such a way we give the explicit form of the one loop renormalization group evolution of the full CKM matrix. Section VI is devoted to the conclusions. 


\section{QUARK YUKAWA COUPLINGS AND CKM MATRIX-HIERARCHY AND EVOLUTION}

The quark and lepton masses in the standard model arise through the Higgs mechanism from the Yukawa couplings which have the following structure in the SM

$$
\sum_{i, j=1}^{3}\left(f_{i j}^{(e)} \bar{e}_{L}^{i} \phi e_{R}^{j}+\left(y_{u}\right)_{i j} \bar{u}_{L}^{i} \widetilde{\phi}_{u_{R}^{j}}^{j}\left(y_{d}\right)_{i j} \bar{d}_{L}^{i} \phi d_{R}^{j}+\text { h.c. }\right) .
$$

Here $f^{(e)}, y_{u}$, and $y_{d}$ are the matrices of the Yukawa couplings of leptons and up and down quarks, respectively. $\phi$ is the scalar Higgs field. The theory itself does not put any restrictions or conditions on the $f^{(e)}, y_{u}$ and $y_{d}$ matrices and from the phenomenological point of view they are constrained by the values of the physical lepton and quark masses

$$
\operatorname{Diag}\left(m_{u}, m_{c}, m_{t}\right)=\left(U_{u}\right)_{L} y_{u}\left(U_{u}\right)_{R}^{\dagger}, \quad \operatorname{Diag}\left(m_{d}, m_{s}, m_{b}\right)=\left(U_{d}\right)_{L} y_{d}\left(U_{d}\right)_{R}^{\dagger}
$$

with diagonal elements being the up and down quark masses after the spontaneous symmetry breaking and the CKM matrix. The diagonalizing matrices $\left(U_{u, d}\right)_{L, R}$ of the biunitary transformations transform the quark fields in Eq. (1) into the physical quark fields. As a consequence the unitary matrix (CKM matrix)

$$
V_{C K M}=\left(U_{u}\right)_{L}\left(U_{d}\right)_{L}^{\dagger}
$$

appears in the charged current.

The observed hierarchy of the quark and lepton masses and the CKM matrix is the following, Refs. [6.7]

$$
\begin{aligned}
& \frac{m_{u}}{m_{c}} \sim \lambda^{4}, \quad \frac{m_{c}}{m_{t}} \sim \lambda^{4}, \quad \frac{m_{d}}{m_{s}} \sim \lambda^{2}, \quad \frac{m_{s}}{m_{b}} \sim \lambda^{2}, \quad \frac{m_{b}}{m_{t}} \sim \lambda^{2}, \quad \frac{m_{e}}{m_{\mu}} \sim \lambda^{4}, \quad \frac{m_{\mu}}{m_{\tau}} \sim \lambda^{2}, \quad \frac{m_{\tau}}{m_{t}} \sim \lambda^{3}, \\
& \left(\begin{array}{ccc}
1-\frac{1}{2} \lambda^{2} & \lambda & \lambda^{3} A(\rho-i \eta) \\
-\lambda & 1-\frac{1}{2} \lambda^{2} & \lambda^{2} A \\
\lambda^{3} A(1-\rho-i \eta) & -\lambda^{2} A & 1
\end{array}\right)
\end{aligned}
$$

with $\lambda \approx 0.22$.

The hypothesis of grand unification assumes that the 3 coupling constants of the standard model converge at the scale $10^{15-16} \mathrm{GeV}$ and the symmetry group of the model becomes larger (e.g. SU(5)). This may also be an origin of the additional symmetries or textures of the QYC at this energy scale. To relate the parameters of the QYC at the GU scale with the observables at low energy one conventionally uses the Renormalization Group Equations (RGE) for the coupling constants and the quark and lepton Yukawa couplings. The structure of the two loop RGE is the following Refs. [1] 3 , 8 ]

$$
\begin{gathered}
\frac{d g_{l}}{d t}=\frac{1}{(4 \pi)^{2}} b_{l} g_{l}^{3}-\frac{1}{(4 \pi)^{4}} G_{l} g_{l}^{3} \\
\frac{d y_{u, d, e, \nu}}{d t}=\left[\frac{1}{(4 \pi)^{2}} \beta_{u, d, e, \nu}^{(1)}+\frac{1}{(4 \pi)^{4}} \beta_{u, d, e, \nu}^{(2)}\right] y_{u, d, e, \nu}
\end{gathered}
$$

The variable $t$ is defined as $t=\ln (E / \mu)$ and the constants $b_{l}$ and functions $G_{l}, \beta_{u, d, e, \nu}^{(1)}$ and $\beta_{u, d, e, \nu}^{(2)}$ are defined for various models in Appendix I. The coefficients $G_{l}, \beta_{u, d, e, \nu}^{(1)}, \beta_{u, d, e, \nu}^{(2)}$ are functions of $g_{l}$ and $y_{u, d, e, \nu}$ so Eqs. (6) form a system of coupled non linear equations and their explicit solution is not known. The Yukawa couplings $y_{u, d, e, \nu}$ are normalized in the following way

$$
\left[y_{u}\right]_{33} \sim 1, \quad\left[y_{d}\right]_{33} \sim \frac{m_{b}}{m_{t}} \sim \lambda^{2}, \quad\left[y_{e}\right]_{33} \sim \frac{m_{\tau}}{m_{t}} \sim \lambda^{3}
$$

The normalization in Eq. (7) is the origin of the hierarchy in Eqs. (6) because the functions $G_{l}, \beta_{u, d, e, \nu}^{(1)}$ and $\beta_{u, d, e, \nu}^{(2)}$ contain the squares and higher powers of $y_{u, d, e, \nu}$.

From Eq. (7) it follows that positive powers of $y_{d, e, \nu}$ are much smaller than the corresponding powers of $y_{u}$. This information enables to find the solution of Eqs. (6) and to study the properties of the solutions. The other origin of the hierarchy in Eqs. (6) is related to the number of loops. Here the inclusion of each additional loop is suppressed by the factor $1 /(4 \pi)^{2} \sim \lambda^{4}$ which is of the same order as $\left(\left[y_{d}\right]_{33}\right)^{2}$ and the correct approximation procedure must take this into account.

Eqs. (6) will be solved and analyzed in the following steps: 
1. Definition of the hierarchy of the equations;

2. Exact solution of the basic equations;

3. Perturbative corrections to the exact solution;

4. Implications of the former results for the quark masses and the CKM matrix

In the rest of the paper we will discuss each step in detail.

Eqs. (6) are the two loop RGE for coupling constants $g_{l}$ and Yukawa couplings $y_{u, d, e, \nu}$. In these equations the terms of different order in $\lambda$ are present. As the first step we will find the approximate form of these equations neglecting all the terms of $\lambda^{4}$ and higher. These approximate equations have the following form

$$
\begin{gathered}
\frac{d g_{l}}{d t}=\frac{1}{(4 \pi)^{2}} b_{l} g_{l}^{3} \\
\frac{d y_{u}}{d t}=\frac{1}{(4 \pi)^{2}}\left[\alpha_{1}^{u}(t)+\alpha_{2}^{u} y_{u} y_{u}^{\dagger}+\alpha_{3}^{u} \operatorname{Tr}\left(y_{u} y_{u}^{\dagger}\right)\right] y_{u}=\frac{1}{(4 \pi)^{2}} E_{1}^{u} y_{u} \\
\frac{d y_{d}}{d t}=\frac{1}{(4 \pi)^{2}}\left[\alpha_{1}^{d}(t)+\alpha_{2}^{d} y_{u} y_{u}^{\dagger}+\alpha_{3}^{d} \operatorname{Tr}\left(y_{u} y_{u}^{\dagger}\right)\right] y_{d}=\frac{1}{(4 \pi)^{2}} E_{1}^{d} y_{d} \\
\frac{d y_{e}}{d t}=\frac{1}{(4 \pi)^{2}}\left[\alpha_{1}^{e}(t)+\alpha_{2}^{e} y_{u} y_{u}^{\dagger}+\alpha_{3}^{e} \operatorname{Tr}\left(y_{u} y_{u}^{\dagger}\right)\right] y_{e}=\frac{1}{(4 \pi)^{2}} E_{1}^{e} y_{e} \\
\frac{d y_{\nu}}{d t}=\frac{1}{(4 \pi)^{2}}\left[\alpha_{1}^{\nu}(t)+\alpha_{2}^{\nu} y_{u} y_{u}^{\dagger}+\alpha_{3}^{\nu} \operatorname{Tr}\left(y_{u} y_{u}^{\dagger}\right)\right] y_{\nu}=\frac{1}{(4 \pi)^{2}} E_{1}^{\nu} y_{\nu}
\end{gathered}
$$

where the values of $\alpha_{i}^{u, d, e, \nu}$ are given in Appendix. Eqs. (8) will be solved exactly and as a next step we will consider Eqs. (6) where the terms of the order $\lambda^{4}$ are kept:

$$
\begin{aligned}
\frac{d g_{l}}{d t} & =\frac{1}{(4 \pi)^{2}} b_{l} g_{l}^{3}-\frac{g_{l}^{3}}{(4 \pi)^{4}}\left(C_{l u} \operatorname{Tr}\left(y_{u} y_{u}^{\dagger}\right)+\sum_{k} b_{k l} g_{k}^{2}\right) \\
\frac{d y_{u}}{d t} & =\frac{1}{(4 \pi)^{2}} E_{1}^{u} y_{u}+\frac{1}{(4 \pi)^{2}}\left[\alpha_{4}^{u} y_{d} y_{d}^{\dagger}+\alpha_{5}^{u} \operatorname{Tr}\left(y_{d} y_{d}^{\dagger}\right)\right] y_{u} \\
& +\frac{1}{(4 \pi)^{4}} F^{u}\left(y_{u} y_{u}^{\dagger}, g_{l}\right) y_{u}=\frac{1}{(4 \pi)^{2}} E_{1}^{u} y_{u}+\frac{1}{(4 \pi)^{4}} E_{2}^{u} y_{u} \\
\frac{d y_{d}}{d t} & =\frac{1}{(4 \pi)^{2}} E_{1}^{d} y_{d}+\frac{1}{(4 \pi)^{2}}\left[\alpha_{4}^{d} y_{d} y_{d}^{\dagger}+\alpha_{5}^{d} \operatorname{Tr}\left(y_{d} y_{d}^{\dagger}\right)\right] y_{d} \\
& +\frac{1}{(4 \pi)^{4}} F^{d}\left(y_{u} y_{u}^{\dagger}, g_{l}\right) y_{d}=\frac{1}{(4 \pi)^{2}} E_{1}^{d} y_{d}+\frac{1}{(4 \pi)^{4}} E_{2}^{d} y_{d} \\
\frac{d y_{e}}{d t} & =\frac{1}{(4 \pi)^{2}} E_{1}^{e} y_{e}+\frac{1}{(4 \pi)^{2}}\left[\alpha_{4}^{e} y_{d} y_{d}^{\dagger}+\alpha_{5}^{e} \operatorname{Tr}\left(y_{d} y_{d}^{\dagger}\right)\right] y_{e} \\
& +\frac{1}{(4 \pi)^{4}} F^{e}\left(y_{u} y_{u}^{\dagger}, g_{l}\right) y_{e}=\frac{1}{(4 \pi)^{2}} E_{1}^{e} y_{e}+\frac{1}{(4 \pi)^{4}} E_{2}^{e} y_{e} \\
& +\frac{1}{(4 \pi)^{4}} F^{\nu}\left(y_{u} y_{u}^{\dagger}, g_{l}\right) y_{\nu}=\frac{1}{(4 \pi)^{2}} E_{1}^{\nu} y_{\nu}+\frac{1}{(4 \pi)^{4}} E_{2}^{\nu} y_{\nu} \\
\frac{d y_{\nu}}{d t} & =\frac{1}{(4 \pi)^{2}} E_{1}^{\nu} y_{\nu}+\frac{1}{(4 \pi)^{2}}\left[\alpha_{4}^{\nu} y_{d} y_{d}^{\dagger}+\alpha_{5}^{\nu} \operatorname{Tr}\left(y_{d} y_{d}^{\dagger}\right)\right] y_{\nu} \\
&
\end{aligned}
$$

The functions $F^{u, d, e, \nu}\left(y_{u} y_{u}^{\dagger}, g_{l}\right)$ are obtained from the functions $\beta_{u, d, e, \nu}^{(2)}$ given in Appendix by putting $y_{d}=y_{e}=0$. Eqs. (9) cannot be explicitly solved but they allow the perturbative solution by transforming them into integral equations. This allows the study of the corrections to the exact solutions of Eqs. (8). 


\section{SOLUTION OF THE RENORMALIZATION GROUP EQUATIONS FOR $y_{u}$ AND $y_{d}$}

The renormalization group equations (6) will be solved in two steps. We will start by solving the one loop equations (8). Eqs. (8a) are very easy to solve and their solution reads

$$
g_{l}(t)=\frac{g_{l}\left(t_{0}\right)}{\sqrt{1-\frac{2 b_{l} g_{l}^{2}\left(t_{0}\right)\left(t-t_{0}\right)}{(4 \pi)^{2}}}}
$$

Eq. (8b) is decoupled from Eqs. (8d) and (8d) and is non linear. Eqs. (8d) and (8d) become linear once $y_{u}-$ the solution of Eq. (8b) is known. We therefore solve Eq. (8b) first.

\section{A. The evolution of $y_{u}$ from one loop RGE}

The crucial fact enabling the exact solution of Eq. $8 \mathrm{~b}$ ) is the observation that the diagonalizing matrices of the biunitary transformation do not depend on the energy. As mentioned before $y_{u}$ can be diagonalized by the biunitary transformation, Eq. (2). The unitary matrices $\left(U_{u}\right)_{L, R}$ are the diagonalizing matrices of the hermitian matrices $H_{u}^{1}=y_{u}(t) y_{u}^{\dagger}(t)$ and $H_{u}^{2}=y_{u}^{\dagger}(t) y_{u}(t)$ and they fulfill the equations which follow from Eq. (8b)

$$
\frac{d}{d t} H_{u}^{i}(t)=\frac{2}{(4 \pi)^{2}}\left\{\alpha_{1}^{u}(t)+\alpha_{2}^{u} H_{u}^{i}+\alpha_{3}^{u} \operatorname{Tr}\left(H_{u}^{i}\right)\right\} H_{u}^{i}, \quad i=1,2 .
$$

The solution of Eq. (11) can be written in the following form

$$
H_{u}^{i}(t)=H_{u}^{i}\left(t_{0}\right)+\left.\sum_{k=1}^{\infty} \frac{\left(t-t_{0}\right)^{k}}{k !} \frac{d^{k} H_{u}^{i}(t)}{d t^{k}}\right|_{t=t_{0}} .
$$

From Eq. (11) one can see that the derivative $\left.\frac{d^{k} H_{u}^{i}}{d t^{k}}\right|_{t=t_{0}}$ is the sum of the powers of $H_{u}^{i}\left(t_{0}\right)$ with scalar coefficients. Thus from the hermiticity of $H_{u}^{i}\left(t_{0}\right)$ it follows that $H_{u}^{i}(t)$ are diagonalized by the same matrices as $H_{u}^{i}\left(t_{0}\right)$ so the diagonalizing matrices $\left(U_{u}\right)_{L}$ and $\left(U_{u}\right)_{R}$ of $H_{u}^{1}(t)$ and $H_{u}^{2}(t)$ are energy independent. Thus the matrix $y_{u}(t)$ has the following representation

$$
y_{u}(t)=\left(U_{u}\right)_{L}^{\dagger} \Delta_{u}(t)\left(U_{u}\right)_{R}
$$

where on the right hand side of Eq. (13) only the diagonal matrix $\Delta_{u}(t)=\operatorname{Diag}\left(m_{u}(t), m_{c}(t), m_{t}(t)\right)$ does depend on $t$ and $\left(U_{u}\right)_{L}$ and $\left(U_{u}\right)_{R}$ are constant matrices that diagonalize $y_{u}\left(t_{0}\right)$. The matrix $\Delta_{u}(t)$ fulfills the following equation

$$
\frac{d}{d t} \Delta_{u}=\frac{1}{(4 \pi)^{2}}\left\{\alpha_{1}^{u}(t)+\alpha_{2}^{u} \Delta_{u}^{2}+\alpha_{3}^{u} \operatorname{Tr}\left(\Delta_{u}^{2}\right)\right\} \Delta_{u}
$$

Eq. (14) splits into 3 equations for the up quark masses

$$
\begin{aligned}
\frac{d m_{u}}{d t} & =\frac{1}{(4 \pi)^{2}}\left\{\alpha_{1}^{u}(t)+\alpha_{3}^{u} m_{t}^{2}\right\} m_{u}, \\
\frac{d m_{c}}{d t} & =\frac{1}{(4 \pi)^{2}}\left\{\alpha_{1}^{u}(t)+\alpha_{3}^{u} m_{t}^{2}\right\} m_{c}, \\
\frac{d m_{t}}{d t} & =\frac{1}{(4 \pi)^{2}}\left\{\alpha_{1}^{u}(t)+\left(\alpha_{2}^{u}+\alpha_{3}^{u}\right) m_{t}^{2}\right\} m_{t} .
\end{aligned}
$$

We first solve Eq. (15c)

$$
m_{t}(t)=\frac{m_{t}\left(t_{0}\right) r_{g}^{1 / 2}(t)}{\sqrt{1-\frac{2}{(4 \pi)^{2}}\left(\alpha_{2}^{u}+\alpha_{3}^{u}\right) m_{t}^{2}\left(t_{0}\right) \int_{t_{0}}^{t} r_{g}(\tau) d \tau}}
$$

where 


$$
r_{g}(t)=\exp \left(\frac{2}{(4 \pi)^{2}} \int_{t_{0}}^{t} \alpha_{1}^{u}(\tau) d \tau\right) .
$$

Eqs. (15a) and $(15 \mathrm{~b})$ are identical and their solution reads

$$
m_{u, c}(t)=m_{u, c}\left(t_{0}\right) r_{g}^{1 / 2}(t) \exp \left(\frac{1}{(4 \pi)^{2}} \alpha_{2}^{u} \int_{t_{0}}^{t} m_{t}^{2}(\tau) d \tau\right) .
$$

The full energy evolution of $y_{u}(t)$ is determined from Eq. (13) using Eqs. (16) and (18).

\section{B. The evolution of $y_{d}$ from one loop RGE}

The one loop evolution of $y_{d}$ is determined from Eq. (8q). The fact that the diagonalizing matrices of $y_{u}(t)$ do not depend on $t$ simplifies Eq. (89) and justifies the following substitution

$$
y_{d}(t)=\left(U_{u}\right)_{L}^{\dagger} W(t)
$$

so $W(t)$ fulfills the equation

$$
\frac{d W}{d t}=\frac{1}{(4 \pi)^{2}}\left\{\alpha_{1}^{d}(t)+\alpha_{2}^{d} \Delta_{u}^{2}+\alpha_{3}^{d} \operatorname{Tr}\left(\Delta_{u}^{2}\right)\right\} W .
$$

The matrix on the right hand side of Eq. (20) has the following form

$$
\begin{aligned}
& \left\{\alpha_{1}^{d}(t)+\alpha_{2}^{d} \Delta_{u}^{2}+\alpha_{3}^{d} \operatorname{Tr}\left(\Delta_{u}^{2}\right)\right\} \\
& =\left(\alpha_{1}^{d}(t)+\alpha_{3}^{d} m_{t}^{2}\right)\left(\begin{array}{lll}
1 & 0 & 0 \\
0 & 1 & 0 \\
0 & 0 & 1
\end{array}\right)+\alpha_{2}^{d} m_{t}^{2}\left(\begin{array}{lll}
0 & 0 & 0 \\
0 & 0 & 0 \\
0 & 0 & 1
\end{array}\right)
\end{aligned}
$$

and the solution $W(t)$ of Eq. (20) is the following

$$
W(t)=\left(r_{g}^{\prime}(t)\right)^{1 / 2} \exp \left(\frac{1}{(4 \pi)^{2}} \alpha_{3}^{d} \int_{t_{0}}^{t} m_{t}^{2}(\tau) d \tau\right) \cdot Z(t) \cdot W\left(t_{0}\right)
$$

where

$$
r_{g}^{\prime}(t)=\exp \left(\frac{2}{(4 \pi)^{2}} \int_{t_{0}}^{t} \alpha_{1}^{d}(\tau) d \tau\right)
$$

and

$$
Z(t)=\left(\begin{array}{ccc}
1 & 0 & 0 \\
0 & 1 & 0 \\
0 & 0 & h(t)
\end{array}\right)
$$

with

$$
h(t)=\exp \left(\frac{1}{(4 \pi)^{2}} \alpha_{2}^{d} \int_{t_{0}}^{t} m_{t}^{2}(\tau) d \tau\right) .
$$

Putting Eqs. (19)-(25) together we obtain the following result for the one loop evolution of $y_{d}(t)$

$$
y_{d}(t)=\left(r_{g}^{\prime}(t)\right)^{1 / 2}(h(t))^{\left(\alpha_{3}^{d} / \alpha_{2}^{d}\right)}\left(U_{u}\right)_{L}^{\dagger} Z(t)\left(U_{u}\right)_{L} y_{d}\left(t_{0}\right) .
$$




\section{Higher order corrections to the $y_{u}$ and $y_{d}$ evolution}

The two loop RGE for $y_{u}$ and $y_{d}$, Eqs. (9b) and (9d) have the following structure

$$
\frac{d y_{u, d}}{d t}=\frac{1}{(4 \pi)^{2}} E_{1}^{u, d} y_{u, d}+\frac{1}{(4 \pi)^{4}} E_{2}^{u, d} y_{u, d}
$$

In Eqs. (27) the terms containing the functions $E_{2}^{u, d}$ are suppressed by the factor $1 /(4 \pi)^{2} \sim \lambda^{4}$ in comparison to the terms with $E_{1}^{u, d}$. Eqs. (27) can be transformed into the integral equations

$$
y_{u, d}(t)=y_{u, d}\left(t_{0}\right)+\frac{1}{(4 \pi)^{2}} \int_{t_{0}}^{t} E_{1}^{u, d} y_{u, d}(\tau) d \tau+\frac{1}{(4 \pi)^{4}} \int_{t_{0}}^{t} E_{2}^{u, d} y_{u, d}(\tau) d \tau
$$

and Eqs. (28) can be transformed into the following recurrence relation

$$
y_{u, d}^{(n)}(t)=y_{u, d}\left(t_{0}\right)+\frac{1}{(4 \pi)^{2}} \int_{t_{0}}^{t} E_{1}^{u, d} y_{u, d}^{(n-1)}(\tau) d \tau+\frac{1}{(4 \pi)^{4}} \int_{t_{0}}^{t} E_{2}^{u, d} y_{u, d}^{(n-1)}(\tau) d \tau .
$$

The functions $y_{u, d}^{(n)}(t)$ converge for $n \rightarrow \infty$ to the solution of Eq. 27) for an arbitrary initial function $y_{u, d}^{(0)}(t)$. If we choose $y_{u, d}^{(0)}(t)$ to be the solution of the one loop RGE, Eqs. (13) and (26) then we obtain

$$
y_{u, d}^{(1)}(t)=y_{u, d}\left(t_{0}\right)+\frac{1}{(4 \pi)^{2}} \int_{t_{0}}^{t} E_{1}^{u, d} y_{u, d}^{(0)} d \tau+\frac{1}{(4 \pi)^{4}} \int_{t_{0}}^{t} E_{2}^{u, d} y_{u, d}^{(0)} d \tau
$$

Now

$$
y_{u, d}\left(t_{0}\right)+\frac{1}{(4 \pi)^{2}} \int_{t_{0}}^{t} E_{1}^{u, d} y_{u, d}^{(0)} d \tau=y_{u, d}^{(0)}(t)
$$

because $y_{u}^{(0)}$ and $y_{d}^{(0)}$ fulfill Eqs. (8b) and (80), respectively. It thus follows that

$$
y_{u, d}^{(1)}(t)=y_{u, d}^{(0)}(t)+\frac{1}{(4 \pi)^{4}} \int_{t_{0}}^{t} E_{2}^{u, d} y_{u, d}^{(0)} d \tau
$$

which means that the lowest order corrections to the solution of the one loop RGE for $y_{u, d}^{(0)}(t)$ are of the relative order $\lambda^{4}$ when compared to the $y_{u, d}^{(0)}(t)$ and Eq. (32) gives the explicit form of this correction.

\section{PROPERTIES OF THE RENORMALIZATION GROUP EVOLUTION OF THE QUARK MASSES AND THE CKM MATRIX}

In this section we will study the physical implications of the results obtained in the previous section. We will present them in the series of the theorems

Theorem 1 The one loop RGEV of the CKM matrix depends on only one function of the energy $h(t)$, given in Eq. (25).

Proof: The CKM matrix, given in Eq. (3) is constructed from the matrices $\left(U_{u}\right)_{L}$ and $\left(U_{d}\right)_{L}$. In section III.A we have shown that $\left(U_{u}\right)_{L}$ does not depend on the energy. The dependence on $t$ can only be contained in $\left(U_{d}\right)_{L}$ which is determined from the matrix $y_{d}(t)$ of the down QYC. The matrices $\left(U_{d}\right)_{L}$ and $\left(U_{d}\right)_{R}$ do not depend on the normalization of $y_{d}(t)$. From Eq. (26) one can see that the only dependence of $y_{d}(t)$ on $t$ apart from the normalization is contained in the matrix $Z(t)$, which is the diagonal matrix and as can be seen from Eq. (24) it depends only on the function $h(t)$. This completes the proof of Theorem 1 .

Theorem 2 The ratios of the down quark masses $m_{d} / m_{s}$ and $m_{s} / m_{b}$ are the functions of only $h(t)$, given in Eq. (25) 
Proof: The down quark masses are obtained after the diagonalization of the down QYC $y_{d}(t)$ and the ratios of $m_{d} / m_{s}$ and $m_{s} / m_{b}$ do not depend on the normalization of $y_{d}(t)$. It thus follows from the proof of Theorem 1 that these ratios are the functions of $h(t)$.

Theorem 3 The ratio of the up quark masses $m_{u} / m_{c}$ is energy independent.

Proof: This theorem follows directly from Eq. (18).

Theorem 4 The next order correction $\rrbracket$ of the RGEV of the CKM matrix are of the order $\lambda^{5}$.

Proof: If we consider RGE in the next order then the terms of the order $\lambda^{4}$ are preserved in the equations and the evolution is governed by Eqs. (9) and the explicit solution in the next leading order is given by Eq. (32). From Eq. (32) one obtains by the direct calculation the following result for the commutators

$$
\begin{aligned}
& {\left[y_{u, d}^{(1)} y_{u, d}^{(1)^{\dagger}}, y_{u, d}^{(0)} y_{u, d}^{(0)^{\dagger}}\right] \sim \lambda^{5},} \\
& {\left[y_{u, d}^{(1)^{\dagger}} y_{u, d}^{(1)}, y_{u, d}^{(0)^{\dagger}} y_{u, d}^{(0)}\right] \sim \lambda^{5} .}
\end{aligned}
$$

From Eqs. (33) using the time independent perturbation theory one obtains that the diagonalizing matrices of the biunitary transformations in the next order are corrected by the terms of the order $\lambda^{5}$ and this completes the proof.

\section{EVOLUTION OF THE CKM MATRIX}

In this section we will find the equations for the one loop evolution of the squares of the absolute values of the off-diagonal elements of the CKM matrix.

The CKM matrix is defined in Eq. (3). Using the fact that $\left(U_{u}\right)_{L}$ is energy independent we obtain from Eq. (26)

$$
\left(U_{u}\right)_{L} y_{d}(t) y_{d}(t)^{\dagger}\left(U_{u}\right)_{L}^{\dagger}=r_{g}^{\prime}(t)(h(t))^{\left(2 \alpha_{3}^{d} / \alpha_{2}^{d}\right)} Z(t)\left(U_{u}\right)_{L} y_{d}\left(t_{0}\right) y_{d}\left(t_{0}\right)^{\dagger}\left(U_{u}\right)_{L}^{\dagger} Z(t)
$$

which can be written in the following way

$$
V_{C K M}(t) M_{d}^{2}(t) V_{C K M}^{\dagger}(t)=r_{g}^{\prime}(t)(h(t))^{\left(2 \alpha_{3}^{d} / \alpha_{2}^{d}\right)} Z(t)\left(U_{u}\right)_{L} y_{d}\left(t_{0}\right) y_{d}\left(t_{0}\right)^{\dagger}\left(U_{u}\right)_{L}^{\dagger} Z(t)
$$

where $M_{d}^{2}(t)$ is the diagonal matrix of the squares of the physical down quarks Yukawa couplings which become the squares of the down quark masses after the spontaneous symmetry breaking. Now differentiating Eq. (35) with respect to $t$ we obtain the following result

$$
\begin{aligned}
& V_{C K M}^{\dagger}(t) \frac{d V_{C K M}}{d t} \\
& =\left(M_{d}^{2}\right)^{-1} V_{C K M}^{\dagger}(t) \frac{d V_{C K M}}{d t} M_{d}^{2}-\left(M_{d}^{2}\right)^{-1} V_{C K M}^{\dagger}(t) \frac{d}{d t}\left(\left(U_{u}\right)_{L} y_{d}(t) y_{d}(t)^{\dagger}\left(U_{u}\right)_{L}^{\dagger}\right) V_{C K M}(t)+\left(M_{d}^{2}\right)^{-1} \frac{d M_{d}^{2}}{d t} .
\end{aligned}
$$

The second term on the right hand side of Eq. (36) is equal

$$
\left(M_{d}^{2}\right)^{-1} V_{C K M}^{\dagger}(t) \frac{d}{d t}\left(\left(U_{u}\right)_{L} y_{d}(t) y_{d}(t)^{\dagger}\left(U_{u}\right)_{L}^{\dagger}\right) V_{C K M}(t)=\frac{d \ln (h)}{d t}\left(\mathbf{R}^{\dagger} \mathbf{R}+\left(M_{d}^{2}\right)^{-1} \mathbf{R}^{\dagger} \mathbf{R} M_{d}^{2}\right)+\frac{d \ln \left(r_{g}^{\prime}(t)(h(t))^{\left(2 \alpha_{3}^{d} / \alpha_{2}^{d}\right)}\right)}{d t} I
$$

where the vector $\mathbf{R}=\left(V_{t d}, V_{t s}, V_{t b}\right)$ and $h(t)$ is given in Eq. (25).

The off diagonal matrix elements of $V_{C K M}^{\dagger}(t) \frac{d V_{C K M}}{d t}$ can now be evaluated from Eq. (36). Using these matrix elements we obtain the following evolution equations

$$
\frac{d\left|V_{u b}\right|^{2}}{d t}=-\frac{h^{\prime}}{h} \frac{m_{d}^{2}+m_{b}^{2}}{m_{d}^{2}-m_{b}^{2}}\left(V_{u b}^{*} V_{u d} V_{t d}^{*} V_{t b}\right)-\frac{h^{\prime}}{h} \frac{m_{s}^{2}+m_{b}^{2}}{m_{s}^{2}-m_{b}^{2}}\left(V_{u b}^{*} V_{u s} V_{t s}^{*} V_{t b}\right)+\text { c.c } \approx-\frac{2 h^{\prime}}{h}\left|V_{u b}\right|^{2}\left|V_{t b}\right|^{2},
$$

\footnotetext{
${ }^{1}$ we include in RGEV equations the terms of the order $\lambda^{4}$ that come from the one loop RGE that contain the terms $y_{d} y_{d}^{\dagger}$ and the two loop contributions of the order $\sim 1$ multiplied by $1 /(4 \pi)^{4}$.
} 


$$
\begin{gathered}
\frac{d\left|V_{c b}\right|^{2}}{d t}=-\frac{h^{\prime}}{h} \frac{m_{d}^{2}+m_{b}^{2}}{m_{d}^{2}-m_{b}^{2}}\left(V_{c b}^{*} V_{c d} V_{t d}^{*} V_{t b}\right)-\frac{h^{\prime}}{h} \frac{m_{s}^{2}+m_{b}^{2}}{m_{s}^{2}-m_{b}^{2}}\left(V_{c b}^{*} V_{c s} V_{t s}^{*} V_{t b}\right)+\text { c.c } \approx-\frac{2 h^{\prime}}{h}\left|V_{c b}\right|^{2}\left|V_{t b}\right|^{2}, \\
\frac{d\left|V_{t d}\right|^{2}}{d t}=-\frac{h^{\prime}}{h} \frac{m_{s}^{2}+m_{d}^{2}}{m_{s}^{2}-m_{d}^{2}}\left(V_{t d}^{*} V_{t s} V_{t s}^{*} V_{t d}\right)-\frac{h^{\prime}}{h} \frac{m_{b}^{2}+m_{d}^{2}}{m_{b}^{2}-m_{d}^{2}}\left(V_{t d}^{*} V_{t b} V_{t b}^{*} V_{t d}\right)+\text { c.c } \approx-\frac{2 h^{\prime}}{h}\left|V_{t d}\right|^{2} \mid\left(1-\left|V_{t d}\right|^{2}\right), \\
\frac{d\left|V_{c d}\right|^{2}}{d t}=-\frac{h^{\prime}}{h} \frac{m_{s}^{2}+m_{d}^{2}}{m_{s}^{2}-m_{d}^{2}}\left(V_{c d}^{*} V_{c s} V_{t s}^{*} V_{t d}\right)-\frac{h^{\prime}}{h} \frac{m_{b}^{2}+m_{d}^{2}}{m_{b}^{2}-m_{d}^{2}}\left(V_{c d}^{*} V_{c b} V_{t b}^{*} V_{t d}\right)+\text { c.c } \approx \frac{2 h^{\prime}}{h}\left|V_{c d}\right|^{2}\left|V_{t d}\right|^{2} .
\end{gathered}
$$

Eqs. (38) can be solved explicitly and the solution reads

$$
\begin{aligned}
& \left|V_{u b}\right|^{2}=\frac{\left|V_{u b}^{0}\right|^{2}}{\left|V_{t b}^{0}\right|^{2}\left(h^{2}-1\right)+1}, \\
& \left|V_{c b}\right|^{2}=\frac{\left|V_{c b}^{0}\right|^{2}}{\left|V_{t b}^{0}\right|^{2}\left(h^{2}-1\right)+1}, \\
& \left|V_{t d}\right|^{2}=\frac{\left|V_{t d}^{0}\right|^{2}}{\left|V_{t d}^{0}\right|^{2}\left(1-h^{2}\right)+h^{2}}, \\
& \left|V_{c d}\right|^{2}=\frac{\left|V_{c d}^{0}\right|^{2}}{\left|V_{t d}^{0}\right|^{2}\left(1-h^{2}\right)+h^{2}} .
\end{aligned}
$$

Here $\left|V_{i j}^{0}\right|^{2}$ are the initial values of the squares of the absolute values of the corresponding CKM matrix elements and $h$ is given in Eq. (25). From the unitarity of the CKM matrix and using Eqs. (39) one can calculate the absolute values of all the remaining matrix elements of the CKM matrix and thus we determine the renormalization group evolution of the full CKM matrix.

\section{CONCLUSIONS}

In this paper we have analyzed the renormalization group evolution of the quark Yukawa couplings and the CKM matrix based on the observed hierarchy of the quark masses and the CKM matrix. The inclusion of the hierarchy greatly simplifies the analysis and leads to simple, explicit results for the evolution of the Yukawa couplings (Eqs. (13) and Eqs. (26)) and the CKM matrix (Eqs. (39)). The other remarkable result is that the diagonalizing matrices of the up quark Yukawa couplings are energy independent in the leading order. This means that the transformation $\left(\psi_{u}\right)_{L, R} \rightarrow\left(U_{u}\right)_{L, R}\left(\psi_{u}\right)_{L, R},\left(\psi_{d}\right)_{L, R} \rightarrow\left(U_{u}\right)_{L, R}\left(\psi_{d}\right)_{L, R}$ will diagonalize the matrix of the up quark Yukawa couplings and it will stay diagonal upon the renormalization group evolution and the CKM matrix will be determined only from the down quarks Yukawa couplings. This fact may simplify the model building based on the symmetries of the quark Yukawa couplings.

\section{ACKNOWLEDGMENTS}

This paper is partially supported by the CoNaCyT (Mexico) project 3512P-E9608. S.R.J.W. gratefully acknowledges partial support by Comisión de Operación y Fomento de Actividades Académicas - COFAA (Instituto Politécnico Nacional). J.G. Mora H. thanks dr. A. Odzijewicz for the hospitality in the Institute of Theoretical Physics of the Białystok University (Poland) during the preparation of the paper. 


\section{APPENDIX: CONSTANTS IN THE RENORMALIZATION GROUP EQUATIONS}

The two loop renormalization group equations for various models have the following structure

$$
\begin{gathered}
\frac{d g_{l}}{d t}=\frac{1}{(4 \pi)^{2}} b_{l} g_{l}^{3}-\frac{1}{(4 \pi)^{4}} G_{l} g_{l}^{3}, \\
\frac{d y_{u, d, e, \nu}}{d t}=\left[\frac{1}{(4 \pi)^{2}} \beta_{u, d, e, \nu}^{(1)}+\frac{1}{(4 \pi)^{4}} \beta_{u, d, e, \nu}^{(2)}\right] y_{u, d, e, \nu}
\end{gathered}
$$

where $b_{l}$ are constants dependent on the model and $G_{l}, \beta_{u, d, e, \nu}^{(1)}$ and $\beta_{u, d, e, \nu}^{(2)}$ are the following functions of the coupling constants and the squares of the Yukawa couplings $H_{u, d, e, \nu}^{(1)}=y_{u, d, e, \nu} y_{u, d, e, \nu}^{\dagger}$

$$
\begin{gathered}
G_{l}=C_{l u} \operatorname{Tr}\left(H_{u}^{(1)}\right)+\sum_{k} b_{k l} g_{k}^{2}, \\
\beta_{l}^{(1)}=\alpha_{1}^{l}(t)+\alpha_{2}^{l} H_{u}^{(1)}+\alpha_{3}^{l} \operatorname{Tr}\left(H_{u}^{(1)}\right)+\alpha_{4}^{l} H_{d}^{(1)}+\alpha_{5}^{l} \operatorname{Tr}\left(H_{d}^{(1)}\right) \\
+\alpha_{6}^{l} H_{e}^{(1)}+\alpha_{7}^{l} \operatorname{Tr}\left(H_{e}^{(1)}\right)+\alpha_{8}^{l} H_{\nu}^{(1)}+\alpha_{9}^{l} \operatorname{Tr}\left(H_{\nu}^{(1)}\right), \\
\beta_{l}^{(2)}=\sum_{l k n} B_{l k n}^{1} g_{k}^{2} g_{n}^{2}+\sum_{l k n} B_{l k n}^{2} g_{k}^{2} H_{n}^{(1)}+\sum_{l k n} B_{l k n}^{3} g_{k}^{2} \operatorname{Tr}\left(H_{n}^{(1)}\right) \\
+\sum_{l k n} B_{l k n}^{4} H_{k}^{(1)} H_{n}^{(1)}+\sum_{l k n} B_{l k n}^{5} H_{k}^{(1)} \operatorname{Tr}\left(H_{n}^{(1)}\right) .
\end{gathered}
$$

The functions $\alpha_{1}^{l}(t)$ are equal

A. for the SM and DHM

$$
\begin{aligned}
& \alpha_{1}^{u}(t)=-\left(\frac{17}{20} g_{1}^{2}+\frac{9}{4} g_{2}^{2}+8 g_{3}^{2}\right) \\
& \alpha_{1}^{d}(t)=-\left(\frac{1}{4} g_{1}^{2}+\frac{9}{4} g_{2}^{2}+8 g_{3}^{2}\right) \\
& \alpha_{1}^{e}(t)=-\left(\frac{9}{20} g_{1}^{2}+\frac{9}{4} g_{2}^{2}\right) \\
& \alpha_{1}^{\nu}(t)=-\left(\frac{9}{20} g_{1}^{2}+\frac{9}{4} g_{2}^{2}\right) .
\end{aligned}
$$

B. for the MSSM

$$
\begin{aligned}
& \alpha_{1}^{u}(t)=-\left(\frac{13}{15} g_{1}^{2}+3 g_{2}^{2}+\frac{16}{3} g_{3}^{2}\right) \\
& \alpha_{1}^{d}(t)=-\left(\frac{7}{15} g_{1}^{2}+3 g_{2}^{2}+\frac{16}{3} g_{3}^{2}\right) \\
& \alpha_{1}^{e}(t)=-\left(\frac{9}{5} g_{1}^{2}+3 g_{2}^{2}\right) \\
& \alpha_{1}^{\nu}(t)=-\left(\frac{3}{5} g_{1}^{2}+3 g_{2}^{2}\right) .
\end{aligned}
$$

The values of the coefficients $b_{l}$ and $\alpha_{k}^{l}$ for the one loop renormalization group equations are given in Tables $\mathbb{\Psi}$, II and III (see e.g. [1 3 ).

TABLE I. Coefficients $b_{l}$ for various models.

\begin{tabular}{lccc}
\hline \hline & $b_{1}$ & $b_{2}$ & $b_{3}$ \\
\hline SM & $\frac{41}{10}$ & $-\frac{19}{6}$ & -7 \\
DHM & $\frac{21}{5}$ & -3 & -7 \\
MSSM & $\frac{33}{5}$ & 1 & -3 \\
\hline \hline
\end{tabular}


TABLE II. The coefficients $\alpha_{k}^{l}$ for various models. The model dependent constants $a, b$ and $c$ are given in Table IIII.

\begin{tabular}{ccccccccc}
\hline \hline$l$ & $\alpha_{2}^{l}$ & $\alpha_{3}^{l}$ & $\alpha_{4}^{l}$ & $\alpha_{5}^{l}$ & $\alpha_{6}^{l}$ & $\alpha_{7}^{l}$ & $\alpha_{8}^{l}$ & $\alpha_{9}^{l}$ \\
\hline$u$ & $\frac{3}{2} b$ & 3 & $\frac{3}{2} c$ & $3 a$ & 0 & $a$ & 0 & 1 \\
$d$ & $\frac{3}{2} c$ & $3 a$ & $\frac{3}{2} b$ & 3 & 0 & 1 & 0 & $a$ \\
$e$ & 0 & $3 a$ & 0 & 3 & $\frac{3}{2} b$ & 1 & $\frac{3}{2} c$ & $a$ \\
$\nu$ & 0 & 3 & 0 & $3 a$ & $\frac{3}{2} c$ & $a$ & $\frac{3}{2} b$ & 1 \\
\hline \hline
\end{tabular}

TABLE III. The constants $a, b$ and $c$ for various models.

\begin{tabular}{lllc}
\hline \hline Model & $a$ & $b$ & $c$ \\
\hline SM & 1 & 1 & -1 \\
DHM & 0 & 1 & $\frac{1}{3}$ \\
MSSM & 0 & 2 & $\frac{2}{3}$ \\
\hline \hline
\end{tabular}

The coefficients for the two loop renormalization group equations that appear in the functions $G_{l}$ and $\beta_{l}^{(2)}$ can be found in Ref. [8].

[1] K. Sasaki, Z. Phys. C 32, 146 (1986).

[2] K.S. Babu, Z. Phys. C 35, 69 (1987); P. Binetruy and P. Ramond, Phys. Lett. B350, 49 (1995).

[3] B. Grzadkowski and M. Lindner, Phys. Lett. B193, 71 (1987); B. Grzadkowski, M. Lindner and S. Theisen, Phys. Lett. B198, 64 (1987).

[4] R.D. Peccei and K. Wang, Phys. Rev. D53, 2712(1996); K. Wang, Phys. Rev. D54, 5750 (1996); H. González et al., Phys. Lett. B 440, 94 (1998); H. González et al., A New symmetry of Quark Yukawa Couplings, page 755, International Europhysics Conference on High Energy Physics, Jerusalem 1997, Eds. Daniel Lellouch, Giora Mikenberg, Eliezer Rabinovici, Springer-Verlag 1999.

[5] S. R. Juárez W., P. Kielanowski and G. Mora, New Properties of the Renormalization Group Equations of the Yukawa Couplings and CKM Matrix in the Proceedings of the Eighth Mexican School Particles and Fields, Oaxaca, México. AIP Conference Proceedings 490 (1998), 351-354, ed. J.C. D’Olivo, G. López C. and M. Mondragón, L.C. Catalog Card No. 99-067150, ISBN 1-56396-895-9, ISSN 0094-243X., DOE CONF-981188.

[6] C. Caso et al., European Physics Journal, C3, 1 (1998).

[7] L. Wolfenstein, Phys. Rev. Lett. 51, 1945 (1983).

[8] H.Arason, et al, Phys. Rev. D46, 3945 (1992). 